\title{
Dermoscopic and Clinical Features of Pigmented Skin Lesions of the Genital Area*
}

\author{
Fatma Pelin Cengiz ${ }^{1}$ \\ Rainer Hofmann Wellenhof ${ }^{2}$
}

\author{
Nazan Emiroglu ${ }^{1}$
}

DOI: http://dx.doi.org/10.1590/abd1806-4841.20153294

\begin{abstract}
BACKGROUND: The dermoscopic features of vulvar melanosis lesions are well known. To our knowledge, there are only a few case reports about dermoscopic features of pigmented genital lesions in male patients.

OвJестіvE: To evaluate dermoscopic and clinical characteristics of benign lesions of the genital area in both males and females, and to assess the distinguishing dermoscopic criteria of vulvar melanosis and atypical melanocytic nevi of the genital type.

METHODS: 68 patients with pigmented genital lesions were included in this observational study (28 male and 40 female). A punch biopsy was taken from all pigmented lesions and histopathological examination was performed on all specimens.

RESULTS: We histopathologically diagnosed: genital melanosis in 40 lesions, atypical melanocytic nevi of the genital type in 15 lesions, melanocytic nevi in 9 lesions, seborrheic keratosis in 4 lesions. The most frequent locations were the glans penis (19 patients, $67.9 \%)$ in males and the labia minora (19 patients, $47.5 \%)$ in females. The mean age of patients with atypical nevi $(28,6 \pm 11,36)$ was significantly lower than the mean age of patients with genital melanosis $(47,07 \pm 15,33)$.

CONCLUSIONS: Parallel pattern is prominent in genital melanosis, ring-like pattern is only observed in genital melanosis. Most pigmented lesions on the genital area are solitary. Blue-white veil and irregular dots are only observed in AMNGT. According to these results, we propose that histopathological examination is performed, especially if blue-white veil and irregular dots are found by dermoscopy.
\end{abstract}

Keywords: Dermoscopy; Melanosis; Nevus, pigmented

\section{INTRODUCTION}

Pigmented lesions of the genital region are uncommon. Its incidence accounts for $10-12 \%$ of the population and for $20 \%$ of all vulvar diseases. ${ }^{1}$ The most frequent locations for pigmented genital lesions are the vulva in women and the glans penis in men. In the literature, there are only a few case reports about dermoscopic features of pigmented genital lesions in male patients. Pigmented skin lesions in the genital area include nevi, melanoma, melanotic macules (lentiginosis, melanosis), angiokeratomas, seborrheic keratosis, squamous cell carcinoma, basal cell carcinoma (BCC).

Postmenopausal women are usually more affected by vulvar melanomas than premenopausal women. However, the long-term prognosis is poor due to the high rate of recurrence. ${ }^{2-6}$ Atypical melanocytic nevi of the genital type (AMNGT) and vulvar melanomas usually affect postmenopausal women and the prognosis is poor due to the high recurrence. ${ }^{2-6}$ Histopathologically atypical melanocytic nevi of the genital type (AMNGT) show similar features to vulvar melanoma. ${ }^{7.8}$

Dermoscopy is a noninvasive tool that helps physicians distinguish melanomas from other pigmented and non-pigmented skin lesions. It gives numerous clues about skin structures in the epidermis, dermoepidermal junction, and dermis. Numerous methods have been developed for the detection of lesion borders. Dermoscopic criteria have been described for the diagnosis of vulvar melanosis, and observational studies have been conducted to define the dermoscopic features of nevi and melanoma on the vulva. ${ }^{9-12}$

\footnotetext{
Received on 29.08.2013.

Approved by the Advisory Board and accepted for publication on 04.03.2014

Financial support: None.

Conflict of interests: None.

Department of dermatology, Bezmialem Vakif University - Istanbul - Turkey.

Medical University of Graz - Graz, Austria.

(C)2015 by Anais Brasileiros de Dermatologia
}

* Study conducted at the Kars State Hospital, Kutahya Tavsanli State Hospital, and Medical University of Graz - Graz, Áustria. 
It may be difficult to clinically and dermatoscopically distinguish between vulvar melanoma and vulvar melanosis. ${ }^{11,13}$ To our knowledge, there have been only a few case reports about dermoscopic features of pigmented genital lesions in male patients. ${ }^{14,15,16,17}$

In this study, we aimed to describe dermoscopic patterns of pigmented lesions in male and female patients, according to the patient's age, and anatomical location and histological subtype of the lesion.

\section{MATERIAL AND METHODS}

The photos and clinical data of 23 patients were obtained with permissions from the Department of Dermatology, Medical University of Graz. The study protocol was approved by the local ethics committee. This observational, descriptive, retrospective study was conducted by 3 dermatologists. 68 patients with pigmented genital lesions were included in the study. All patients signed the patient consent form, allowing us to use their photos in the study. Inclusion criteria were: older than 18 years of age, consultation in the department of dermatology due to a pigmented lesion of the genital area. For all participants, sex, age, and dermoscopic patterns, location, diameter and histological subtype of the lesion were recorded. Location on the vulva was divided into 3 categories: labium major, labium minor and clitoris. Location on the penis was divided into 3 categories: glans penis, meatus and shaft of the penis. Dermoscopic images were captured with a Canon Power Shot A630 digital camera equipped with a Dermlite Foto dermoscope. Solitary lesions were excised for histopathological examination and a $4 \mathrm{~mm}$ punch biopsy was performed in lesions larger than $10 \mathrm{~mm}$. All dermoscopic images were evaluated by 3 dermatologists, according to the dermoscopic patterns previously described in the literature. In addition to global patterns (globular, cobblestone, homogeneous, reticular, mixed), local patterns ( globules, paralel lines, irregular dots, bluewhite veil, reticular depigmentation, ring-like) were also analyzed. ${ }^{9,10,12,18-21}$ Based on the dermatopathological diagnosis, the 68 pigmented lesions were then classified as vulvar melanosis, melanocytic nevus, atypical nevus and seborrheic keratosis.

SPSS 15.0 (SPSS Inc., Chicago, IL, U.S.A.) was used in the statistical analysis. Statistical significance was considered as $p<0.05$. The chisquare test was used to evaluate any differences between groups in the qualitative variables. For correlations between variables, Spearman correlation coefficients were estimated.

\section{RESULTS \\ General Results}

A total of 68 pigmented genital lesions from 68 patients were included ( 28 male and 40 female) in the study. The mean age of the males was $40.67 \pm 13.50$ years and the mean age of the females was $38.25 \pm$ 16.83. The 68 pigmented lesions consisted of: 40 melanosis $(58.8 \%), 15$ atypical nevi $(22.1 \%), 9$ melanocytic nevi $(13.2 \%)$, and 4 seborrheic keratosis $(5.9 \%)$, histopathologically (Table 1$)$. The mean diameter of lesions was $8.41 \pm 4.60 \mathrm{~mm}$. There was no statistical difference between males and females regarding the diameter of lesions $(p=0,112)$ (Table 1$)$.

The most frequent location for pigmented lesions in males was the glans penis (19 patients, $67.9 \%$ ), followed by the meatus (6 patients, $21.4 \%)$, and the shaft of the penis (3 patients, 10.7\%). In females, the most common site was the labia minora (19 patients, $47.5 \%$ ), followed by the labia majora (18 patients, $45.0 \%$ ), and both the labia majora and minora (3 patients, $7.5 \%)$.

TAble 1: Patients' Clinical Data and Skin Lesions

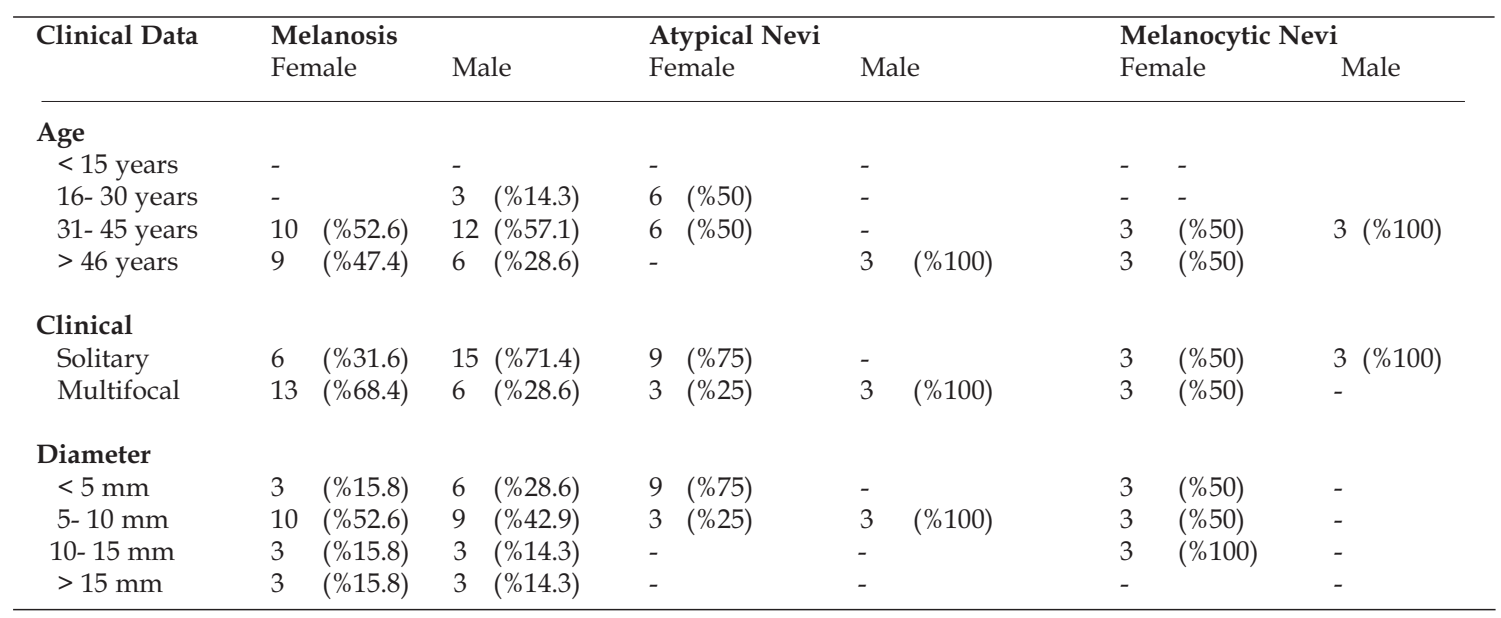


Dermoscopic Patterns and Clinical Characteristics of Melanosis

The mean age for melanosis was $47.07 \pm 15.33$ years. 21 patients $(52.5 \%)$ with melanosis were male and 19 were female $(47.5 \%) .21$ lesions $(52.5 \%)$ were solitary, $19(47.5 \%)$ were multifocal. Lesions consisted of 25 cases of melanosis with two colors $(62.5 \%), 12$ cases of melanosis with one color (30\%), and 3 cases of melanosis with three colors $(7.5 \%)$. The mean diameter was $9.77 \pm 5.13 \mathrm{~mm}$. The most common location in males was the glans penis (12 patients, $57.1 \%$ ), followed by the meatus (6 patients, $28.5 \%$ ) and the shaft of the penis ( 3 patients, $14.2 \%$ ). The labia minora was the most frequent site (13 patients, $68.4 \%$ ) in females. Melanosis on the labia majora, and both on the labia majora and minora were observed in 3 patients (15.8\%) each.

The most frequent pattern was the parallel pattern (21 patients, 52.5\%). The globular pattern was present in 13 melanosis lesions (32.5\%). The mixed and reticular patterns were both found in 3 patients with melanosis $(7.5 \%)$. The ring-like pattern was present in 20 melanosis lesions (50\%). We found the ringlike pattern in 10 melanosis lesions located on the glans penis $(50 \%), 3$ on the shaft of the penis $(15 \%)$ and 7 on the labia minora (35\%). The ring-like pattern was observed in 12 multifocal melanosis lesions (60\%) and in 8 solitary melanosis lesions (40\%). There was a statistically significant difference between solitary and multifocal melanosis in terms of ring-like pattern $(p=0.005)$ (Table 2) (Figures 1 and 2).

\section{Dermoscopic Patterns and Clinical Characteristics of Atypical Nevi}

The mean age for atypical nevi was $28.6 \pm 11.36$. 3 patients (20\%) with atypical nevi were male and 12 were female (80\%). 9 atypical nevi $(60 \%)$ were solitary and 6 were multifocal ( $40 \%) .9$ atypical nevi $(60 \%)$ had two colors, 3 had one color $(20 \%)$ and $3(20 \%)$ had three colors. The mean diameter was $5.20 \pm 0.77 \mathrm{~mm}$. All atypical nevi in males were observed on the glans penis ( 3 patients). In females, 6 of them were located on the labia minora and 6 on the labia majora.

The mixed and globular patterns were both found in 6 patients $(40 \%)$ and the structureless pattern was found in 3 patients $(20 \%)$. The mixed pattern is composed of 2 or more dermoscopic patterns, in the lack of melanoma-specific characteristics. The most common combination was that of the parallel pattern with linear and curvilinear brown streaks with the globular pattern with globules and dots. Irregular dots were observed in 9 patients $(60 \%)$. The blue-white veil was present in 2 patients (13\%). The ring-like pattern was not observed in atypical nevi (Figure 3).

\section{Dermoscopic Patterns and Clinical Characteristics of Melanocytic Nevi}

The mean age for melanocytic nevi was $39.33 \pm$ 10.14. 3 patients $(33.3 \%)$ with melanocytic nevi were male and 6 were female (66.7\%). 6 melanocytic nevi $(66.7 \%)$ were solitary and 3 were multifocal (33.3\%). 9 melanocytic nevi $(100 \%)$ had 2 colors. The mean diameter was $8.33 \pm 4.27 \mathrm{~mm}$. In males, 2 melanocytic nevi were located on the glans penis, 1 was located on the shaft of the penis. In females, 4 were located on the labia majora $(66.6 \%)$ and 2 on the labia minora $(33.4 \%)$.

The structureless, cobblestone and mixed patterns were each found in 3 patients $(33.3 \%)$. The ringlike pattern was not observed in melanocytic nevi. Milia- like cysts were found in 2 melanocytic nevi (22.2\%) (Figure 4).

\section{Dermoscopic Patterns and Clinical Characteristics of Seborrheic Keratosis}

The mean age for seborrheic keratosis was 29.73 \pm 8.35. All patients with seborrheic keratosis were female and all lesions were located on the labia majo-

TAble 2: Dermoscopic Features of Pigmented Lesions

\begin{tabular}{|c|c|c|c|}
\hline \multirow{2}{*}{$\begin{array}{l}\text { Dermoscopic Features } \\
\text { Global Pattern } \\
\text { Parallel Pattern }\end{array}$} & Melanosis & Atypical Nevi & Melanocytic Nevi \\
\hline & $(\% 52.5)$ & - & - \\
\hline Structureless Pattern & - & $(\% 20)$ & $(\%$ 33.3) \\
\hline Cobblestone Pattern & - & - & $(\%$ 33.3) \\
\hline Globular Pattern & $13 \quad(\%$ 32.5) & $(\% 40)$ & - \\
\hline Reticular Pattern & $3 \quad(\% 7.5)$ & - & - \\
\hline Mixed Pattern & $(\% 7.5)$ & $(\% 40)$ & (\% 33.3) \\
\hline Local Pattern & & & \\
\hline Ring-like Pattern & $20 \quad(\% 50)$ & - & - \\
\hline Blue-white Veil & - & $(\% 13)$ & - \\
\hline Milia-like Cysts & - & - & $(\%$ 22.2) \\
\hline Irregular Dots & - & $(\% 60)$ & $(70-2.2)$ \\
\hline Reticular Depigmentation & - & - & - \\
\hline
\end{tabular}




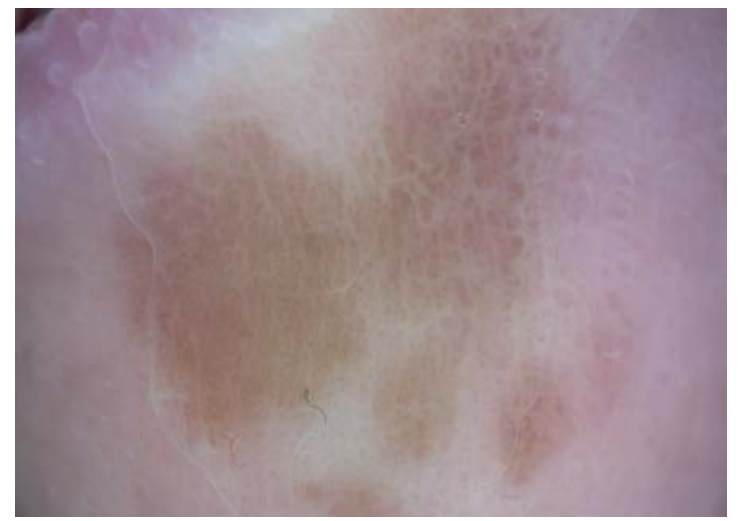

Figure 1: Melanosis on the glans penis showing a globular pattern

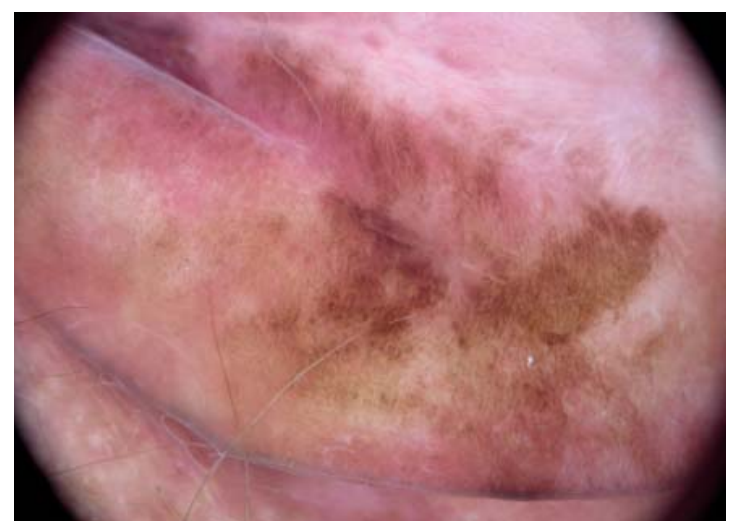

FIGURE 2: Melanosis on the labia minora showing a parallel pattern (uniform linear and curved structures)

ra. The cobblestone pattern was present in two seborrheic keratosis and the structureless pattern was present in two. Among the other nonmelanocytic lesions, comedo-like openings were found in 2 of 4 seborrheic keratosis. Milia- like cysts were present in one of them. The ring-like pattern was not observed in seborrheic keratosis.

The mean age of patients with atypical nevi $(28.6 \pm 11.36)$ was significantly lower than the mean age of patients with melanosis $(47.07 \pm 15.33)$. The mean diameter of melanosis lesions $(9.77 \pm 5.13)$ was significantly larger than the mean diameter of atypical nevi lesions $(5.20 \pm 0.77)(p<0.001)$. There was a positive correlation between diameter of lesion and melanosis $(p<0.005, r=0.354)$. Additionally, there was a positive correlation between the number of colors and the female gender $(p<0.001, r=0.461)$. There was a negative correlation between the presence of the ring-like pattern and the female gender $(\mathrm{p}<0.001, \mathrm{r}=$ 0.505).

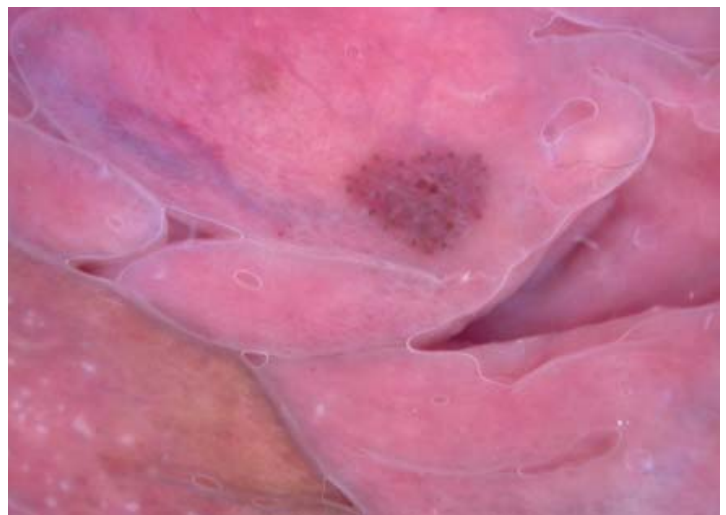

Figure 3: Atypical melanocytic nevus of the genital type. Irregular dots on the periphery of the lesion

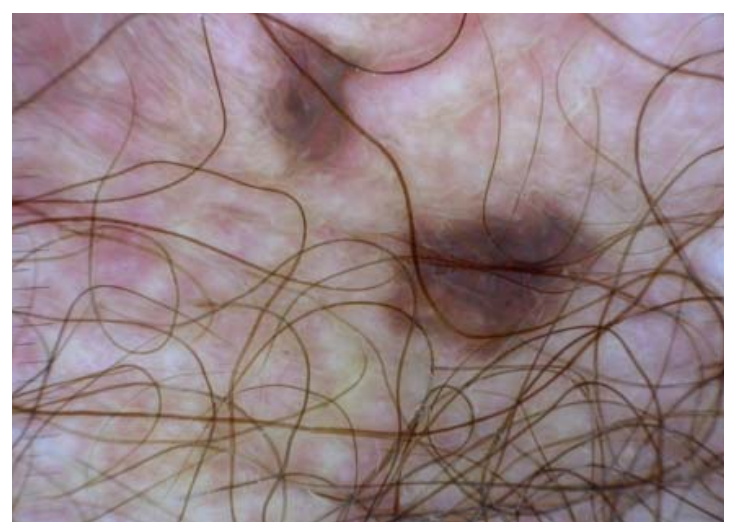

FIGURE 4: Common melanocytic nevus on the labia majora showing a structureless pattern

\section{DISCUSSION}

Pigmented skin lesions of the vulva are present in $12-19 \%$ of the women who have had gynecological examination. ${ }^{9}$ Vulvar melanosis is the most frequent lesion among these pigmented disorders. ${ }^{22}$ Vulvar melanosis usually occurs in white women and has an unknown etiology. Even though vulvar melanosis has a benign prognosis, it causes concern to the patient and to the physician, owing to its melanoma-like presentation. ${ }^{11.13}$

Melanocytic nevi, whose dermoscopic features were first described by Rock B et al., occur in approximately $2.3 \%$ of females. ${ }^{22}$ Atypical melanocytic nevi of the genital type (AMNGT) may histopathologically mimic malignant melanoma by the presence of atypical cells (melanocytes with larger, pleomorphic nuclei, sometimes with prominent nucleoli).

In this study, we evaluated only benign lesions of the genital area, including genital melanosis, atypical melanocytic nevi of the genital type (AMNGT), 
melanocytic nevi and seborrheic keratosis.

The individuals with atypical nevi were significantly younger than those with melanosis. This result is consistent with those of previous studies. ${ }^{12}$ In addition to the solitary presence of most genital melanosis lesions, atypical and melanocytic nevi were also present as solitary lesions. About one fourth of the lesions observed in this study were atypical melanocytic nevi of the genital type (AMNGT), whose diagnosis was confirmed by histopathological examination. In our study, the number of AMNGT was higher than in previous studies. This result may be related to the high female-to-male ratio noticed in previous studies. ${ }^{7,8,11} \mathrm{In}$ our study, the female-to-male ratio was lower than the one found in previous studies. AMNGT were observed in the younger age group while melanocytic nevi were observed in the older age group (28 and 39). Our results support the findings by Ferrari et al. In the analysis of dermoscopic features of 16 vulvar melanocytic nevi, Ronger-Savle et al showed that the homogeneous and globular pattern is the most frequent dermoscopic pattern. ${ }^{12}$ According to the results from Ferrari et al., the globular pattern and the mixed pattern were the most common patterns in pigmented lesions of the vulva. ${ }^{11}$ In our case series, most AMNGT had a mixed or globular pattern. These data are similar to those reported by Ferrari and colleagues.

The mixed pattern, which is created by parallel lines together with homogeneous pigmentation or globules, is more commonly present in atypical nevi than in melanosis and melanocytic nevi. Ferrari et al. suggested that a pigmented lesion must be followed up or excised if it has a mixed pattern. ${ }^{11}$ Our data supports this suggestion. Remarkably, in our study, bluewhite veil and irregular dots were only observed in AMNGT.

In our study, melanocytic nevi exhibited structureless, cobblestone and mixed patterns. Virgili et al showed that homogeneous and globular patterns were commonly seen in melanocytic nevi, in which a homogeneous brown-gray pigmentation and shades of brown, respectively, appeared. ${ }^{23}$ These data are similar to those reported by Virgili and colleagues. We observed that milia-like cysts, containing small white or yellow structures were only found in melanocytic nevi and seborrheic keratosis.

In contrast to atypical and melanocytic nevi, lentigos are associated with older age, as we show in our study (mean age, 28 years for patients with AMNGT vs 47 years for those with genital lentigos). It is reported that lentigos (melanosis) are the most common pigmented lesions among women, present in approximately $7 \%$ of females., ${ }^{9,22}$ Vulvar melanoma is an uncommon tumor, with an incidence of $0.10 / 100.000$ women annually and it accounts for
$2-10 \%$ of all vulvar malignancies. ${ }^{4}$ In the literature, it was suggested that vulvar melanoma has a peak incidence after the 6th decade., ${ }^{2,3}$ Ferrari et al reported the disappearence of 2 melanocytic nevi in two young girls in a 4-year dermoscopic follow-up. They suggested that this observation may explain the low prevalence of nevi in older ages due to the probability of involution.

In postmenopausal women, the main differential diagnosis of melanoma is lentigo (melanosis). Vulvar melanosis may occur as multifocal macules with irregular shape, which usually show all the criteria of $A B C D$ rules. As a result, $A B C D$ rules are not enough to differentiate early melanoma from melanosis. Mannone et al observed the structureless pattern, which is histopathologically characterized by moderate to evident hyperpigmentation along the basal cell layer as the most common pattern in vulvar melanosis in their series. ${ }^{9}$ In a larger case series, globular-like and parallel, reticular-like, cobblestone-like and ring-like patterns, and their relation with multifocality were also described in melanosis. ${ }^{10}$ In our study, the most typical pattern of melanosis in genital localization is the 'parallel pattern'. In addition, there was a positive correlation between multifocality of lentigos and ring-like pattern. This result is consistent with Ferrari et al. ${ }^{10}$

To date, 17 cases of vulvar melanoma have been reported in the literature. ${ }^{11,19,23-27}$ In these cases, a multicomponent pattern composed by blue-white veil, atypical network, irregular streaks, dots and atypical vessels was exhibited. Ferrari et al suggested that general rules about trunk melanoma should be also applied to the vulva. According to the results of Ferrari et al and previous series, none of the vulvar melanomas showed globular pattern. In addition, all the vulvar melanomas in their case series had 4 points or more when the algorithm of Ronger-Savle et al was applied to the lesions.

It has also been reported that reticular depigmentation, a white network consisting of white, fragile lines, is a highly valuable dermoscopic finding in early vulvar melanomas. ${ }^{11,28}$ This dermoscopic feature had been associated with melanoma and Spitz nevi. ${ }^{18,28}$ Reticular depigmentation was not observed in our case series. Due to the benign histopathological results of our cases, our data support previous findings.

Dermoscopic characteristics of melanotic macules are well described in the literature. Uniform brown color (structureless or homogeneous pattern), parallel (linear and curved streaks) pattern (the most common pattern in our study), ring-like pattern (multiple round to oval structures with well-defined regular borders) are the terms used to define mucosal melanosis by dermoscopy. In our study, we also 
observed globular and reticular patterns in melanosis lesions. Blue-white veil may be found in melanosis, even though this feature is more common in melanoma. ${ }^{9,10}$ As a result, vulvar pigmented lesions dermoscopically exhibiting blue-white veil should be excised or biopsed to rule out melanoma.

The limitation in our study were the low sample size and the inclusion of only benign lesions. The parallel pattern was the most frequent pattern in melanosis. Additionally, ring-like pattern was only observed in melanosis. Lesions had 2 or more standardized dermoscopic colours in females. The ringlike pattern was more common in males than females. Most pigmented lesions on the genital area were solitary lesions. Blue-white veil and irregular dots were only observed in AMNGT lesions. Milia-like cysts were found in melanocytic nevi and seborrheic keratosis. Even if there is currently no available informa- tion whether vulvar melanoma arise from AMNGT or not, it is better to remove lesions. Clues for AMNGT are given by dermoscopy.

\section{CONCLUSION}

In the literature, there are data on dermoscopic and clinical features of vulvar lesions. To our knowledge, our study is the first study that evaluated dermoscopic and clinical characteristics of both female and male patients. We aimed to determine specific dermoscopic features of AMNGT and lesions which must be followed-up. It may be difficult to distinguish between benign and malign lesions by dermoscopy, hence, all suspicious lesions must be removed. Further prospective studies of a larger number of patients and genital pigmented lesions will give more definite results.

\title{
REFERENCES
}

1. Hosler GA, Moresi JM, Barrett TL. Naevi with site-related atypia: a review of melanocytic naevi with atypical histological features based on anatomic site. J Cutan Pathol. 2008;35:889-98.

2. 2. Irvin WP Jr, Legallo RL, Stoler MH, Rice LW, Taylor PT Jr, Andersen WA. Vulvar melanoma: a retrospective analysis and literature review. Gynecol Oncol. 2001;83:457-65.

3. Wechter ME, Gruber SB, Haefner HK, Lowe L, Schwartz JL, Reynolds KR, et al. Vulvar melanoma: a report of 20 cases and a review of the literature. J Am Acad Dermatol. 2004;50:554-62.

4. Lotem M, Anteby S, Peretz T, Ingber A, Avinoach I, Prus D. Mucosal melanoma of the female genital tract is a multifocal disorder. Gynecol Oncol. 2003;88:45-50.

5. Ragnarsson-Olding BK. Primary malignant melanoma of the vulva - an aggressive tumor for modeling the genesis of non-UV light-associated melanomas. Acta Oncol. 2004;43:421-35.

6. De Simone P, Silipo V, Buccini P, Mariani G, Marenda S, Eibenschutz L, et al. Vulvar melanoma: a report of 10 cases and a review of the literature. Melanoma Res. 2008:18:127-33.

7. Gleason BC, Hirsch MS, Nucci MR, Schmidt BA, Zembowicz A, Mihm MC Jr, et al. Atypical genital nevi: a clinicopathologic analysis of 56 cases. Am J Surg Pathol. 2008:32:51-7.

8. Ribè A. Melanocytic lesions of the genital area with attention given to atypical genital nevi. J Cutan Pathol. 2008:35:24-7.

9. Mannone F, De Giorgi V, Cattaneo A, Massi D, De Magnis A, Carli P. Dermoscopic features of mucosal melanosis. Dermatol Surg. 2004;30:1118-23.

10. Ferrari A, Buccini $P$, Covello $R$, De Simone $P$, Silipo V, Mariani $G$, et al. The ringlike pattern in vulvar melanosis: a new dermoscopic clue for diagnosis. Arch Dermatol. 2008;144:1030-4.

11. Ferrari A, Zalaudek I, Argenziano G, Buccini P, De Simone P, Silipo V, et al. Dermoscopy of pigmented lesions of the vulva: a retrospective morphological study. Dermatology. 2011;222:157-66.

12. Ronger-Savle $S$, Julien V, Duru G, Raudrant D, Dalle S, Thomas L. Features of pigmented vulval lesions on dermoscopy. Br J Dermatol. 2011;164:54-61.

13. Acikgöz G, Demirci CC, Arca E. Vulvanın Pigmente Lezyonları. Turk J Dermatol. 2012; 6:39-44.

14. Coras-Stepanek B, von Portatius A, Dyall-Smith D, Stolz W. Dermatoscopy of pigmented extramammary Paget disease simulating melanoma. J Am Acad Dermatol. 2012;67:e144-6.

15. Ishioka P, Yamada S, Michalany NS, Hirata SH. Dermoscopy of Bowen's disease: pigmented variant on the penis. An Bras Dermatol. 2012;87:482-4.

16. Betti R, Menni S, Crosti C. Melanoma of the glans penis. Eur J Dermatol. 2005;15:113-5.

17. de Giorgi V, Massi D, Brunasso G, Salvini C, Mastrolorenzo A, Zuccati G, et al. Eruptive multiple blue nevi of the penis: a clinical dermoscopic pathologic case study. J Cutan Pathol. 2004;31:185-8.

18. Argenziano G, Soyer HP, Chimenti S, Talamini R, Corona R, Sera F, et al. Dermoscopy of pigmented skin lesions: results of a consensus meeting via the internet. J Am Acad Dermatol. 2003:48:679-93.

19. de Giorgi V, Massi D, Salvini C, Mannone F, Cattaneo A, Carli P. Thin melanoma of the vulva: a clinical, dermoscopic-pathologic case study. Arch Dermatol. 2005;141:1046-7.

20. Soyer HP, Argenziano G, Chimenti S, Ruocco V. Dermoscopy of pigmented skin lesions. Eur J Dermatol. 2001;11:270-6.

21. Argenziano G, Soyer HP, de Giorgi V, Piccolo D, Carli P, Delfino M, et al. Interactive Atlas on Dermoscopy. Milan: EDRA Medical Publishing and New Media; 2001.

22. Rock B, Hood AF, Rock JA. Prospective study of vulvar nevi. J Am Acad Dermatol. 1990;22:104-6.

23. Virgili A, Zampino MR, Corazza M. Primary vulvar melanoma with satellite metastasis: dermoscopic findings. Dermatology. 2004;208:145-8.

24. Lin J, Koga H. Takata M, Saida T. Dermoscopy of pigmented lesions on mucocutaneous junction and mucous membrane. Br J Dermatol. 2009;161:1255-61.

25. Stolz W, Braun-Falco O, Bilek P, Landthaler M, Burgdorf WHC, Cognetta AB. Pigmented lesions on the mucosa. In: Stolz W, Braun- Falco O, Bilek P, Landthaler M, Burgdorf WHC, Cognetta AB, editors. Color Atlas of Dermoscopy. 2nd ed. Berlin: Blackwell Publishing; 2002. P. 151-4.

26. Puig S, Malvehy J. Criterios dermatoscopicos de las lesiones pigmentadas en las mucosas. In: Malvehy J, Puig S, editors. Principios de dermatoscopia. Barcelona: CEGE; 2009. p 32132.

27. Wolf $\mid H$. Melanocytic nevi on the genitalia and other melanocytic nevi in other locations. In: Soyer HP, Argenziano G, Hofmann-Wellenhof R, Johr R, editors. Color Atlas of Melanocytic Lesions of the Skin. Berlin: Springer; 2007. P. 119-23.

28. Lozzi GP, Piccolo D, Micantonio T, Altamura D, Peris K. Early melanomas dermoscopically characterized by reticular depigmentation. Arch Dermatol 2007;143:808-9.

\author{
MAILING ADDRESS: \\ Fatma Pelin Cengiz \\ Department of dermatology \\ Bezmialem Vakif University - Istanbul - Turkey \\ E-mail:fpelinozgen@hotmail.com
}

How to cite this article: Cengiz FP, Emiroglu N, Hofmann-Wellenhof R. Dermoscopic and Clinical Features of Pigmented Skin Lesions of the Genital Area. An Bras Dermatol. 2015;90(2):178-83. 